\title{
Arterial Spin Labeling-Can Neurointerventionists Rely on This Novel Technique? A Case Series on Successful Use of Arterial Spin Labeling in Acute Stroke Imaging
}

\author{
K. R. Rahul ${ }^{1} \quad$ P. Santhosh ${ }^{1} \quad$ M. P. Dilip Kumar ${ }^{2}$ Pankaj Mehta ${ }^{2}$ Mathew Cherian ${ }^{2}$
}

${ }^{1}$ Division of Neuro and Vascular Interventional Radiology, Department of Radiology, Kovai Medical Centre and Hospital, Coimbatore, Tamil Nadu, India

${ }^{2}$ Division of Interventional Radiology, Department of Radiology, Kovai Medical Centre and Hospital, Coimbatore, Tamil Nadu, India

J Clin Interv Radiol ISVIR 2017;1:144-149.

\author{
Address for correspondence Mathew Cherian, MD, PDCC, Division of \\ Interventional Radiology, Department of Radiology, Kovai Medical \\ Centre and Hospital, Coimbatore, Tamil Nadu, India \\ (e-mail: dr.mathewcherian@gmail.com).
}

\begin{abstract}
Presence of ischemic penumbra is the principal factor that decides the need for mechanical thrombectomy in acute stroke patients with large vessel occlusion. Our objective was to evaluate the usefulness of arterial spin labeling (ASL) in detecting diffusion perfusion mismatch and directing patients into mechanical thrombectomy. We retrospectively studied all patients with acute nonhemorrhagic stroke in the anterior circulation, who had undergone stroke imaging with ASL followed by mechanical thrombectomy from July 2016 to November 2016. Area of diffusion perfusion mismatch was graded semiquantitatively into three grades: small, medium, and large. Mismatch was compared with 30-day modified Rankin scale (mRS) score.

\section{Keywords}

- arterial spin labeling

- perfusion imaging

- diffusion perfusion mismatch

- modified Rankin scale score

Interpretable PASL-perfusion images were obtained in all patients. Diffusion perfusion mismatches were present in all patients. Out of six patients with good mRS score, five patients had large diffusion perfusion mismatch. Two out of three patients with poor mRS were secondary to failed recanalization, in spite of large mismatch. One out of nine patients had poor outcome as well as a small area of mismatch. ASL is a rapid noninvasive imaging technique in acute stroke that has got the potential to detect ischemic penumbra.
\end{abstract}

\section{Introduction}

Conventional imaging of stroke was mainly dependent on computed tomography (CT), which included noncontrast imaging that assessed the ASPECTS (Alberta Stroke Program Early CT Score), contrast CT angiography, and CT perfusion. Though these techniques continue to be the mainstay in stroke imaging, there are certain disadvantages such as high radiation dose and need of contrast administration. Further, interpretation of the images can be difficult and, at times,

received

January 16, 2017

accepted after revision

May 1, 2017

published online

September 1, 2017 impossible if the patient becomes noncooperative during the contrast study. Considering these issues, there is a need for an alternative imaging technique that gives the same information without contrast administration or radiation. Magnetic resonance imaging (MRI) sequences that include diffusion imaging, noncontrast perfusion arterial spin labeling (ASL) can probably have a distinct advantage. We present the successful use of ASL perfusion imaging in selecting patients for mechanical thrombectomy and the outcome.
Copyright $(2017$ by Indian Society of Vascular and Interventional Radiology
License terms

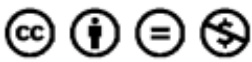



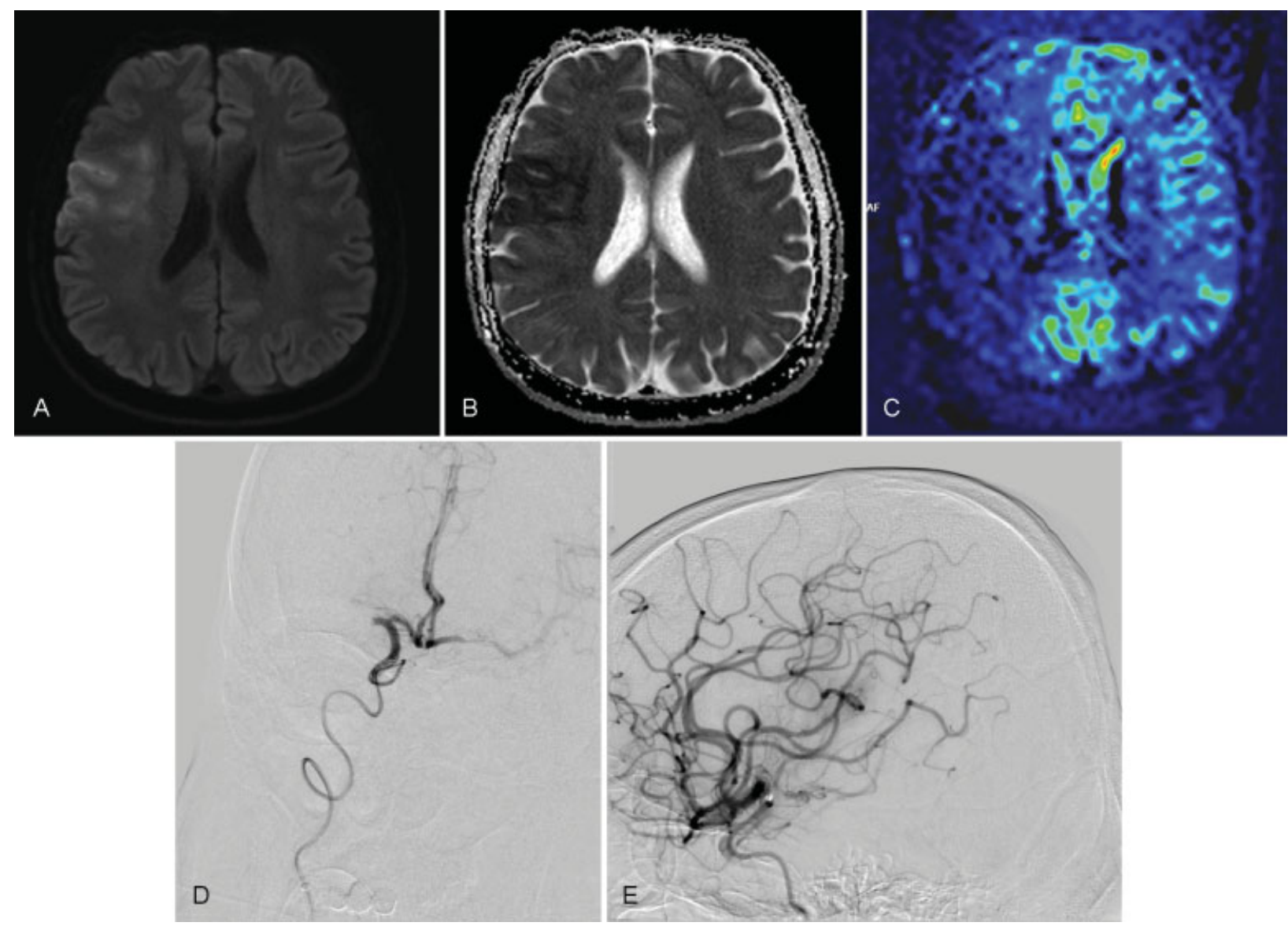

Fig. 1 A 48-year-old man with acute left hemiplegia; MRI showed large diffusion perfusion mismatch; 30-day mRS 2. (A) Small area of diffusion restriction. (B) Corresponding ADC image. (C) ASL color map shows large mismatch. (D) Cerebral angiogram showing right middle cerebral artery M1 segment occlusion. (E) Reperfused middle cerebral artery following mechanical thrombectomy.

\section{Background}

Current treatment protocol for nonhemorrhagic stroke advocates intravenous thrombolysis with tPA (tissue plasminogen activator) if the patient comes within window period of 3 hours (class 1 recommendation) ${ }^{1}$ and can be extended to 4.5 hours with some precautions. If there is a large vessel occlusion in CT angiography with at least $50 \%$ or more cortical vessels seen retrogradely filling via collaterals or magnetic resonance angiography with large diffusion-perfusion mismatch, the patient will benefit from mechanical thrombectomy-the window period for mechanical thrombectomy can go up to 6 hours, according to recent trials. ${ }^{2}$

Following an acute infarct, the volume of the viable tissue, which carries a high risk of ischemia termed the "ischemic penumbra," determines the benefit of reperfusion. Ischemic penumbra is the tissue that is still structurally intact (not infarcted) and hence viable but electrically dysfunctional due to ischemia. This area of the brain can be salvaged if we intervene and reperfuse within the window period. ${ }^{3}$

The penumbra is the salvageable area of the ischaemic brain. It is evaluated by performing a diffusion scan which shows the irreversibly damaged tissue and a perfusion scan which shows the threatened but not dead brain parenchyma. The difference between the two is termed perfusion deficit (termed as "diffusion-perfusion mismatch") indicates ischemic penumbra. ${ }^{4}$

However, MRI may not be freely available; further, at times performing an MRI may be impractical, and if we need to depend solely on CT for acute stroke imaging, the ASPECTS score and CT angiogram source images ${ }^{5}$ can give an estimate of the area already infarcted- similar to MR DWI. Collateral score predicts the ischemic penumbra and final infarct volume. The collateral score is measured in multiphase CT angiography, by comparing the retrograde filling of the cortical arteries on the side of large vessel occlusion with similar arteries in the opposite unaffected hemisphere. It is graded from 0 (no collateral supply) to 3 (complete collateral supply). Grade 1 denotes collaterals filling $\leq 50 \%$ but $>0 \%$ of the occluded vessel territory. A score 2 indicates collaterals $>50 \%$ but $<100 \%$ of the occluded vessel territory. A score 3 is given for $100 \%$ collateral supply. ${ }^{6}$

Poor collateral score carries a major risk for developing a near-complete vascular territory infarct. In other words, when the CT angiogram collaterals are good, the area of infarct will be less even if the territory is not reperfused. Souza et $\mathrm{al}^{7}$ reported a negative correlation between collateral score and volume of DWI infarct core. In short, a good collateral score is equivalent to a large ischemic penumbra. 

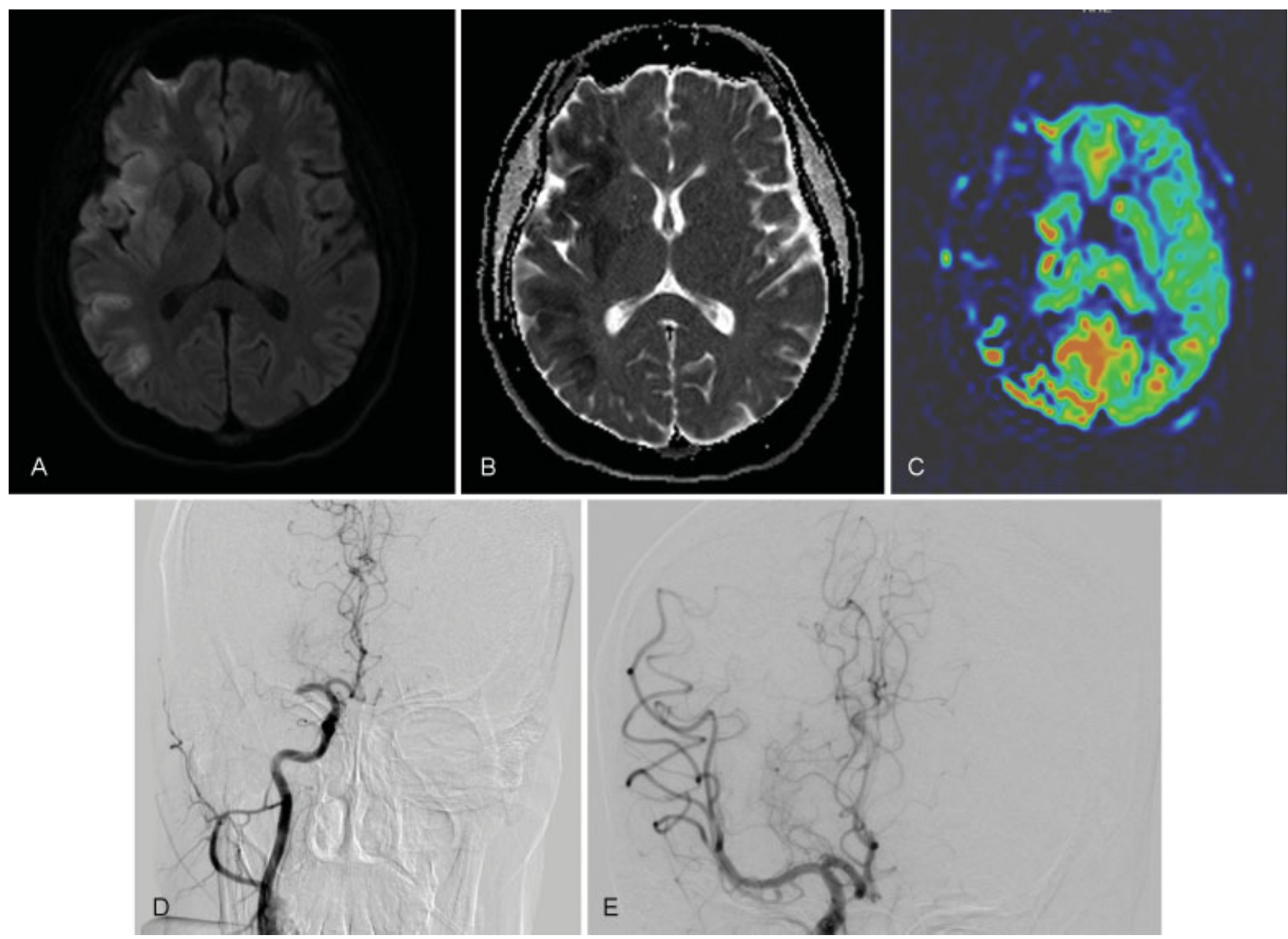

Fig. 2 A 40-year-old woman with acute left hemiplegia; MRI showed small diffusion perfusion mismatch; 30-day mRS 3. (A) Large area of diffusion restriction. (B) Corresponding ADC image. (C) ASL color map shows small mismatch. (D) Cerebral angiogram showing right middle cerebral artery M1 segment occlusion. (E) Reperfused middle cerebral artery following mechanical thrombectomy.

CT perfusion also has the capability of demonstrating penumbra. However, it has not gained widespread acceptance in the management of stroke. The major drawback to CT angiogram and perfusion includes ionizing radiation and the possibility of completely losing all diagnostic quality images if the patient becomes noncooperative during the examination. Variability in the accuracy and reliability of perfusion CT, due to different methods used for postprocessing to derive at infarct core and penumbra, is yet another drawback. ${ }^{8,9}$

The primary goal of perfusion imaging in acute stroke is to identify tissue that can be salvaged with appropriate therapy and also to decide the lack of benefit if a patient with no mismatch is treated.

\section{Magnetic Resonance Perfusion Imaging}

Perfusion imaging is usually done with contrast. However, current MRI machines offer diagnostic quality noncontrast techniques for perfusion. Different MRI techniques are available for cerebral perfusion measurements in routine clinical practice.
1. DSC (dynamic susceptibility contrast) MRI

2. DCE (dynamic contrast enhanced) MRI

3. ASL (arterial spin labeling)-noncontrast technique

Contrast-enhanced dynamic susceptibility (DSC) T2*weighted technique is the conventional technique. Approximately $20 \mathrm{~mL}$ of gadolinium is injected at 4 to $6 \mathrm{~mL} / \mathrm{s}$. and an echo planar sequence is used to acquire whole-brain coverage with $\geq 12$ slices during a 90 - to 120 -second acquisition time. A nonlinear signal loss will be produced by the flow of paramagnetic contrast agent through the cerebral circulation due to the contrast susceptibility or T2* effect. Tissue signal changes produced by this $\mathrm{T}^{*}$ effect are used to create the time-to-signal intensity curve. Color- or intensity-coded hemodynamic maps can be generated from either raw data or processed data. ${ }^{10}$

Dynamic contrast-enhanced MRI is the other exogenous contrast-based method. DCE-MRI uses rapid and repeated T1-weighted images to measure the signal changes induced by the paramagnetic tracer in the tissue as a function of time. ${ }^{11}$

ASL technique is a technique that does not require a contrast agent but rather utilizes the spins of endogenous water protons as a tracer. Thus endogenous arterial water is 

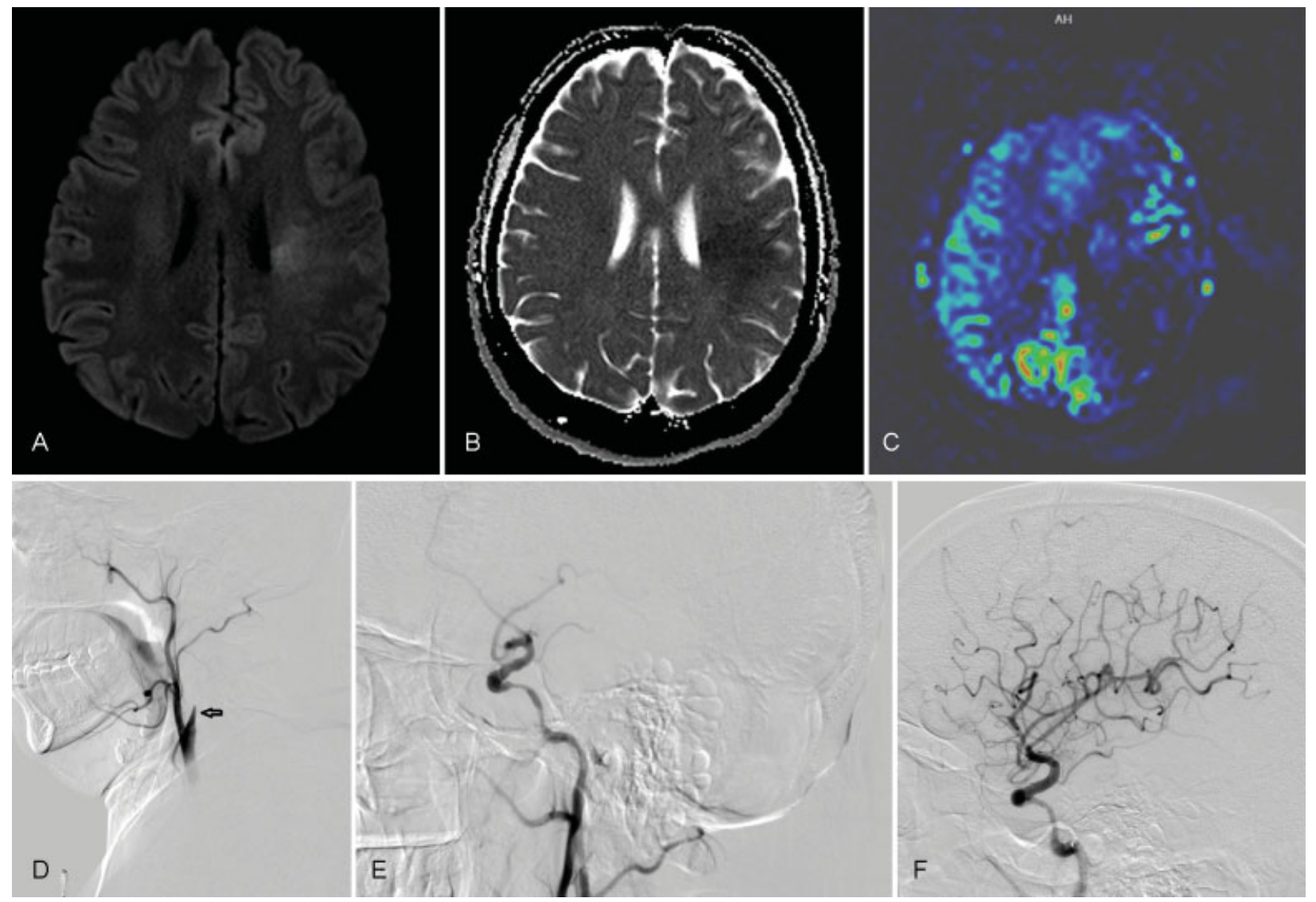

Fig. 3 A 46-year-old man with acute right hemiplegia; MRI showed large diffusion perfusion mismatch; 30-day mRS 1. (A) Small area of diffusion restriction. (B) Corresponding ADC image. (C) ASL color map shows large mismatch. (D, E) Cerebral angiogram showing left internal carotid artery (arrow) and middle cerebral artery M1 segment occlusion. (F) Reperfused middle cerebral artery following internal carotid artery stenting and mechanical thrombectomy.

electromagnetically labeled and used as a freely diffusible tracer.

There are three broad types of labeling methods for ASL:

1. Pulsed ASL (PASL)

2. Continuous ASL (CASL)

3. Pseudo-continuous ASL (pCASL)

In PASL, the application of a short radiofrequency pulse, tags arterial blood flowing upstream by inverting the spin polarity of protons flowing into the imaging plane. Another technique known as the CASL method utilizes a continuous radiofrequency (RF) of weak intensity to label blood upstream of the slice. In pCASL, the third technique, continuous labeling is achieved by a train of rapidly repeating low-tip RF pulses and alternating sign (bipolar) magnetic field gradients.

General advantages of ASL techniques include the ability to assess the entire brain, lack of radiation, and avoidance of contrast with ASL as well as the detailed information provided when combined with other MRI modalities such as DWI and magnetic resonance angiography. We can repeat the study even if the patient moves during the procedure, as there is no contrast involved such as CT

The drawbacks to perfusion MRI currently include lack of quantitative measurements, marginally longer examination time compared with CT, decreased sensitivity to hemorrhages, and its limitation in patients with a contraindication to magnetic fields.

\section{Patients and Methods}

\section{Patient Population}

We retrospectively studied all patients with acute nonhemorrhagic stroke in the anterior circulation, who had undergone stroke imaging with ASL followed by mechanical thrombectomy from July 2016 to November 2016.

Exclusion criteria included hemorrhagic stroke, patients with ischemic stroke who had not undergone mechanical thrombectomy, all posterior circulation stroke, and patients who had undergone mechanical thrombectomy without MRI to reasons such as contraindication for MRI or medically unstable patients.

NIHSS (National Institute of Health Stroke Scale) at admission and 30-day modified Rankin scale (mRS) were measured in all patients.

Our institute's stroke protocol contains CT imaging of the brain to rule out intracerebral hemorrhage. Ischemic stroke patients within a window period 6 hours will be subjected to immediate MRI. The magnetic resonance protocol includes four 
sequences: DWI, perfusion imaging using ASL, noncontrast time-of-flight (ToF) angiogram of the brain, and susceptibilityweighted imaging (SWI). Visual inspection for large vessel occlusion, perfusion deficits, and perfusion-diffusion mismatches was performed. Mismatch was graded as large, medium, and small. If the diffusion-restricted area represents $<25 \%$, of the area of perfusion deficit, it is considered as large mismatch. If the diffusion-restricted area measures 25 to $50 \%$ and $>50 \%$ of perfusion deficit, mismatch is considered medium and small, respectively. All patients with a large ischemic penumbra (diffusion perfusion mismatch) were considered candidates for mechanical thrombectomy.

\section{Arterial Spin Labeling Protocol}

ASL was performed in all patients with a Siemens 3T machine (MAGNETOM Skyra, Siemens Healthcare, Erlangen, Germany) using the pulsed technique. Total acquisition time was 3 minutes 5 seconds.

The protocol used was FAIR Q2TIPS labeling, TR 5,000 milliseconds, TE 15.72 milliseconds, slice thickness $4 \mathrm{~mm}$, distance factor 50\%, flip angle 180 degrees; 32 slices, $256 \mathrm{~mm}$ FOV, base resolution 64, phase resolution 98.

\section{Results}

Nine patients (eight males and one female) of anterior circulation stroke underwent magnetic resonance perfusion imaging followed by mechanical thrombectomy. The patients' age ranged from 40 to 64 years (median age: 51 years). NIHSS score ranged from 7 to 24 with a median score of 17 .

Interpretable PASL-perfusion images were obtained in all patients. Significant perfusion-diffusion mismatches were present in all patients.

Five out of nine patients (-Figs. 1-3) had isolated M1 segment occlusion, and out of these, three had successful recanalization. In two patients, middle cerebral artery (MCA) recanalization was not achieved, out of whom one had an underlying stenosis of M1 segment with an evidence of old infarct on CT. Two of the nine cases had internal carotid artery (cervical segment) occlusion at the origin with associated occlusion in M1 segment. Both the patients had successful recanalization with simultaneous stenting of carotid artery. One patient had dissection of cervical segment of internal carotid artery along with M1 segment occlusion. He required stenting of proximal internal carotid artery with recanalization of MCA. The last case had stenosis of internal carotid artery with $\mathrm{M} 1$ segment occlusion. This patient did not require a stent because the response to angioplasty was adequate. The M1 segment was treated by suction thrombectomy.

Thirty-day mRS scores were as follows: one patient had a poor score of 4 (patient with underlying stenosis in first part of MCA that did not open after three attempts of mechanical thrombectomy); a score of 3 was noted in two patients, out of these, one patient had a small diffusion-perfusion mismatch and the other had a large mismatch with M1 occlusion, which could not be recanalized; and mRS score of 0 to 2 (functional independence) was achieved in six patients, and five of these patients showed large diffusion perfusion mismatch ( - Table $\mathbf{1}$ ).

\section{Discussion}

The aim of stroke therapy revolves around salvaging ischemic tissue before they get infarcted. Tissue that has been permanently damaged cannot be revived. Current imaging strategies are directed toward finding out the volume of tissue that is permanently damaged vis-a-vis the volume that is ischemic. Detecting the presence of a diffusion-perfusion mismatch in acute stroke with large vessel occlusion is very important as these are the patients who will benefit from mechanical thrombectomy and their outcome in terms of mRS score will be better. Currently, CT perfusion or contrast magnetic resonance perfusion are considered the gold standard in showing ischemic penumbra. Noncontrast magnetic resonance techniques have got inherent disadvantages and were considered less useful. However, current sequences on 3T systems are consistently able to reproduce excellent quality perfusion images using ASL technique. ${ }^{12,13}$

Noncontrast magnetic resonance perfusion studies in all our patients were of good interpretable quality, and the final outcome in all of them corresponded closely to the expected

Table 1 Thirty-day mRS scores

\begin{tabular}{|l|l|l|l|l|l|l|l|}
\hline Patient No. & $\begin{array}{l}\text { Window } \\
\text { period (h) }\end{array}$ & $\begin{array}{l}\text { NIHSS } \\
\text { score }\end{array}$ & $\begin{array}{l}\text { Large vessel } \\
\text { occluded }\end{array}$ & $\begin{array}{l}\text { Grade of } \\
\text { mismatch }\end{array}$ & $\begin{array}{l}\text { Recanalization } \\
\text { achieved or not }\end{array}$ & 30-d mRS & $\begin{array}{l}\text { Additional } \\
\text { Information }\end{array}$ \\
\hline 1 & 4.5 & 20 & M1 & Large & No & 4 & Stenosis of M1 MCA \\
\hline 2 & 4 & 18 & M1 & Large & Yes & 2 & - \\
\hline 3 & 3 & 16 & ICA, M1 & Large & Yes & 2 & ICA stenting done \\
\hline 4 & 3.5 & 20 & M1 & Medium & Yes & 1 & - \\
\hline 5 & 4 & 15 & ICA, M1 & Large & Yes & 2 & ICA stenting done \\
\hline 6 & 4 & 24 & M1 & Large & No & 3 & Failed recanalization \\
\hline 7 & 3 & 7 & ICA stenosis, M1 & Large & Yes & 1 & ICA angioplasty done \\
\hline 8 & 4.5 & 22 & M1 & Small & Yes & 3 & - \\
\hline 9 & 1.5 & 14 & ICA dissection, M1 & Large & Yes & 1 & ICA stenting done \\
\hline
\end{tabular}

Abbreviation: ICA, internal carotid artery; M1 MCA, M1 segment of middle cerebral artery; mRS, modified Rankin scale. 
recovery based on the penumbra whenever the vessel was recanalized and corresponded to the studies performed by Hartkamp et al and Bivard et al. ${ }^{14,15}$ This study demonstrates that ASL is successful in demonstrating the ischemic penumbra.

In our study, $66.6 \%$ (six out of nine) of our patients had functional independence as shown by mRS score of 0 to 2 . Out of these, five patients (83\%) had large diffusion-perfusion mismatch. A poor mRS score was noted in one patient with small ischemic penumbra demonstrated by ASL. Two patients had poor outcome in spite of large ischemic penumbra but only due to failure to achieve recanalization of the occluded vessel.

Further, PASL perfusion imaging provides rapid noninvasive multislice imaging in acute ischemic stroke, as the study takes approximately 3 minutes and can be easily repeated because there is no contrast if patients' motion resulted in poor-quality images, whereas in CT angiography, repeating the study will involve an additional 40 to $60 \mathrm{~mL}$ contrast and additional radiation. In elderly patients, large volumes of contrast can be detrimental especially because additional contrast will be required during endovascular procedure. Further in emergency situation, renal function is never tested and a patient with low glomerular filtration rate (GFR) could end up with further deterioration in renal function.

The total time for the current MRI protocol is 12 minutes that included DWI, ASL, FLAIR (fluid-attenuated inversion recovery), and cerebral and neck vessel angiogram, which gives a complete anatomy and perfusion dynamics of stroke. The main disadvantage of MRI is its nonavailability and the lack of arterial spin labeling in most currently available machines because this is a new software. However, all newgeneration MRIs have the capability of performing arterial spin labeling.

\section{Limitations of the Study}

The major limitation is the small size of the study population; further, it is not a case-control study. Considering that all patients were cases of acute stroke in which speed was crucial to salvage the brain, ASL was not compared with another control such as CT perfusion/angiography or magnetic resonance contrast perfusion.

\section{Conclusion}

ASL is a rapid noninvasive imaging technique in acute stroke that can consistently demonstrate the penumbra when combined with diffusion imaging. Thus it has the potential to replace CT perfusion in the current guidelines for stroke. However, further studies are required to validate its role as the first line imaging for stroke therapy. When combined with other sequence, MRI could be the one-stop imaging solution for stroke assessment.

\section{References}

1 Powers WJ, Derdeyn CP, Biller J, et al; American Heart Association Stroke Council. 2015 American Heart Association/American Stroke Association Focused Update of the 2013 Guidelines for the Early Management of Patients with Acute Ischemic Stroke Regarding Endovascular Treatment: A Guideline for Healthcare Professionals from the American Heart Association/American Stroke Association. Stroke 2015;46(10):3020-3035

2 Saver JL, Goyal M, Bonafe A, et al; SWIFT PRIME Investigators. Stent-retriever thrombectomy after intravenous t-PA vs. t-PA alone in stroke. N Engl J Med 2015;372(24):2285-2295

3 Astrup J, Siesjö BK, Symon L. Thresholds in cerebral ischemia-the ischemic penumbra. Stroke 1981;12(06):723-725

4 Kidwell CS, Alger JR, Saver JL. Evolving paradigms in neuroimaging of the ischemic penumbra. Stroke 2004;35(11, Suppl 1):2662-2665

5 Camargo ECS, Furie KL, Singhal AB, et al. Acute brain infarct: detection and delineation with $\mathrm{CT}$ angiographic source images versus nonenhanced CT scans. Radiology 2007;244(02):541-548

6 Tan IYL, Demchuk AM, Hopyan J, et al. CT angiography clot burden score and collateral score: correlation with clinical and radiologic outcomes in acute middle cerebral artery infarct. AJNR Am J Neuroradiol 2009;30(03):525-531

7 Souza LCS, Yoo AJ, Chaudhry ZA, et al. Malignant CTA collateral profile is highly specific for large admission DWI infarct core and poor outcome in acute stroke. AJNR Am J Neuroradiol 2012;33 (07):1331-1336

8 Kudo K, Sasaki M, Ogasawara K, Terae S, Ehara S, Shirato H. Difference in tracer delay-induced effect among deconvolution algorithms in CT perfusion analysis: quantitative evaluation with digital phantoms. Radiology 2009;251(01):241-249

9 Bivard A, Levi C, Spratt N, Parsons M. Perfusion CT in acute stroke: a comprehensive analysis of infarct and penumbra. Radiology 2013;267(02):543-550

10 Copen WA, Schaefer PW, Wu O. MR perfusion imaging in acute ischemic stroke. Neuroimaging Clin N Am 2011;21(02):259-283, X

11 Jahng G-H, Li K-L, Ostergaard L, Calamante F. Perfusion magnetic resonance imaging: a comprehensive update on principles and techniques. Korean J Radiol 2014;15(05):554-577

12 Niibo T, Ohta H, Yonenaga K, Ikushima I, Miyata S, Takeshima H. Arterial spin-labeled perfusion imaging to predict mismatch in acute ischemic stroke. Stroke 2013;44(09):2601-2603

13 Guo L, Zhang Q Ding L, et al. Pseudo-continuous arterial spin labeling quantifies cerebral blood flow in patients with acute ischemic stroke and chronic lacunar stroke. Clin Neurol Neurosurg 2014;125:229-236

14 Hartkamp NS, van Osch MJP, Kappelle J, Bokkers RPH. Arterial spin labeling magnetic resonance perfusion imaging in cerebral ischemia. Curr Opin Neurol 2014;27(01):42-53

15 Bivard A, Krishnamurthy V, Stanwell P, et al. Arterial spin labeling versus bolus-tracking perfusion in hyperacute stroke. Stroke 2014;45(01):127-133 